\title{
On homogeneous Lagrange means
}

\author{
Janusz Matkowski
}

Published online: 20 May 2014

(C) The Author(s) 2014. This article is published with open access at Springerlink.com

\begin{abstract}
Let $f$ be a real differentiable function in an open interval $I$ with one-to-one derivative. We observe that if the Lagrange mean $L^{[f]}$ of a generator $f$ is conditionally positively homogeneous, then $f$ must be of the class $C^{\infty}$ and the function

$$
g(x):=x f^{\prime}(x)-f(x), \quad x \in I,
$$

is also a generator of $L^{[f]}$ i.e. that $L^{[g]}=L^{[f]}$. We show that this fact and a result on equality of two Lagrange means allow easily to determine all positively homogeneous Lagrange means.
\end{abstract}

Keywords Mean · Lagrange mean · Homogeneous mean · Equality of means · Differential equation

Mathematics Subject Classification Primary: 26A24 - 26E60 $\cdot 39 \mathrm{~B} 22$

\section{Introduction}

Let $f$ be a real differentiable function defined in an open interval $I \subset \mathbb{R}$. By the Lagrange mean-value theorem, there exists a two-place function $L^{[f]}: I^{2} \rightarrow I$, called a Lagrange mean of a generator $f$, such that, for all $x, y \in I$,

$$
f(x)-f(y)=f^{\prime}\left(L^{[f]}(x, y)\right)(x-y) .
$$

If the derivative $f^{\prime}$ is one-to-one, then the mean $L^{[f]}$ is uniquely determined.

It is well known that if the Lagrange mean $L^{[f]}$ is positively homogeneous, then it is a generalized logarithmic mean $\mathcal{L}^{[p]}([4,5] \mathrm{cf}$. also [3], p. 404, Theorem 6). The main purpose

J. Matkowski $(\varangle)$

Faculty of Mathematics, Computer Science and Econometrics,

University of Zielona Góra,

ul Prof. Z. Szafrana 5a, 65-516 Zielona Góra, Poland

e-mail: Matkowski@wmie.uz.zgora.pl 
of this note is to present a new proof of this fact. The idea is based on the observation that any generator $f$ of a conditionally positively homogeneous Lagrange mean $L^{[f]}: I^{2} \rightarrow I$ must be of the class $C^{\infty}$ and that the function

$$
g(x):=x f^{\prime}(x)-f(x), \quad x \in I,
$$

is also a generator of $L^{[f]}$, that is $L^{[g]}=L^{[f]}$. These facts and a result on equality of two Lagrange means (cf. [1], [6]) imply that

$$
x f^{\prime}(x)-f(x)=a f(x)+b x+c, \quad x \in I,
$$

for some $a, b, c \in \mathbb{R}$. Solving this differential equation we obtain all the homogeneous Lagrange means.

In section 2 we recall the definitions of a mean, the Lagrange mean based on Lagrange's mean-value theorem (that is used in this paper), its integral counterpart and some of its properties. In section 3 we prove Theorem 3.2, a slight modification of the results on the equality of two Lagrange means (cf. [1] and [6]). In the first part of Theorem 3.2 we assume that at least one of two equal means is uniquely determined. In section 4 we show that any generator $f$ of the conditionally homogeneous Lagrange mean $L^{[f]}$ in an open interval $I$, that is such that, for all $x, y \in I$ and $t>0$,

$$
t x, t y \in I \Longrightarrow L^{[f]}(t x, t y)=t L^{[f]}(x, y),
$$

is infinitely times differentiable (Theorem 4.1). Applying these results, in section 5 we determine all the conditionally homogeneous Lagrange means (Theorem 5.1).

Applicability of the method to determine the form of all conditionally homogeneous Cauchy means is discussed in the last section.

\section{Means, Lagrange means and some their properties}

Let $I \subset \mathbb{R}$ be an interval. A function $M: I^{2} \rightarrow \mathbb{R}$ is said to be a mean in $I$ if

$$
\min (x, y) \leq M(x, y) \leq \max (x, y), \quad x, y \in I .
$$

A mean $M$ is called strict if these inequalities are sharp for all $x, y \in I, x \neq y$; and symmetric if $M(x, y)=M(y, x)$ for all $x, y \in I$.

Remark 2.1 If $M: I^{2} \rightarrow \mathbb{R}$ is a mean in an interval $I$, then

(1) $M$ is reflexive, that is

$$
M(x, x)=x, \quad x \in I
$$

(2) for any interval $J \subset I$,

$$
M\left(J^{2}\right)=J
$$

in particular, $M\left(I^{2}\right)=I$.

Remark 2.2 If $M: I^{2} \rightarrow \mathbb{R}$ is a reflexive function, that is if $M(x, x)=x$ for all $x \in I$, and (strictly) increasing with respect to each variable, then it is a (strict) mean in $I$.

Remark 2.3 If $M: I^{2} \rightarrow I$ is a continuous and strict mean in $I$, then for any $u \in$ int $I$ there are $x, y \in$ int $I, x \neq y$, such that $M(x, y)=u$. 
By the Lagrange mean-value theorem, if a real function $f$ defined on an interval $I \subset \mathbb{R}$ is differentiable, then there exists a mean $L^{[f]}: I^{2} \rightarrow I$ such that, for all $x, y \in I, x \neq y$,

$$
\frac{f(x)-f(y)}{x-y}=f^{\prime}\left(L^{[f]}(x, y)\right) .
$$

If moreover $f^{\prime}$ is one-to-one, then

$$
L^{[f]}(x, y)=\left\{\begin{array}{ll}
\left(f^{\prime}\right)^{-1}\left(\frac{f(x)-f(y)}{x-y}\right) & \text { for } x \neq y \\
x & \text { for } x=y
\end{array},\right.
$$

so $L^{[f]}$ is uniquely determined and, by the Darboux property of derivative, $f^{\prime}$ is continuous and strictly monotonic (cf. [7], Remark 2.1), so $f$ is either strictly convex or strictly concave in $I$.

The mean $L^{[f]}$ is called the Lagrange mean and the function $f$ its generator. It obvious that $L^{[f]}$ is continuous, symmetric and strict, that is

$$
\min (x, y)<L^{[f]}(x, y)<\max (x, y), \quad x, y \in I, x \neq y .
$$

Remark 2.4 Assume that $h: I \rightarrow \mathbb{R}$ is continuous and strictly monotonic. Then $\mathcal{L}^{h}: I^{2} \rightarrow I$ defined by

$$
\mathcal{L}^{h}(x, y):= \begin{cases}h^{-1}\left(\frac{1}{y-x} \int_{x}^{y} h(t) d t\right) & \text { for } x \neq y \\ x & \text { for } x=y\end{cases}
$$

is a strict mean in the interval $I$ and it is called a Lagrange mean (cf. for instance [1], [2], [3]). Setting $h:=f^{\prime}$, it is not difficult to see that $\mathcal{L}^{h}=L^{[f]}$. Note that in the integral definition of the Lagrange mean $\mathcal{L}^{h}$ from the very beginning it is assumed that the function $h$ (the derivative of $f$ ) is continuous and strictly monotonic.

\section{Equality of two Lagrange means}

We need the following [cf. [6], Theorem 3.2]

Lemma 3.1 Let $I \subset \mathbb{R}$ be an open interval, $f, g: I \rightarrow \mathbb{R}$, and let $I_{f}$ stand for the range of the two variable function

$$
(x, y) \longrightarrow \frac{f(x)-f(y)}{x-y}, \quad x, y \in I, x \neq y .
$$

Suppose that $f$ is strictly convex or strictly concave on I. If $\phi: I_{f} \rightarrow \mathbb{R}$ satisfies the functional equation

$$
\frac{g(x)-g(y)}{x-y}=\phi\left(\frac{f(x)-f(y)}{x-y}\right), \quad x, y \in I, x \neq y,
$$

then there exist $a, b \in \mathbb{R}$ such that

$$
\phi(u)=a u+b, \quad u \in I_{f} .
$$

Applying this lemma we prove the following (cf. [6]) 
Theorem 3.2 Let $I \subset \mathbb{R}$ be an interval. Suppose that the functions $f, g: I \rightarrow \mathbb{R}$ are differentiable.

(i) If $f^{\prime}$ is one-to-one and

$$
L^{[g]}=L^{[f]}
$$

then there are $a, b, c \in \mathbb{R}$, such that

$$
g(x)=a f(x)+b x+c, \quad x \in I .
$$

(ii) If $f^{\prime}$ and $g^{\prime}$ are one-to-one and

$$
L^{[g]}=L^{[f]}
$$

then there are $a, b, c \in \mathbb{R}, a \neq 0$, such that

$$
g(x)=a f(x)+b x+c, \quad x \in I .
$$

Proof To prove (i) assume that $f^{\prime}$ is one-to-one and $L^{[g]}=L^{[f]}$. Since

$$
g^{\prime}\left(L^{[g]}(x, y)\right)=\frac{g(x)-g(y)}{x-y}, \quad x, y \in I, x \neq y,
$$

and

$$
L^{[g]}(x, y)=L^{[f]}(x, y)=\left(f^{\prime}\right)^{-1}\left(\frac{f(x)-f(y)}{x-y}\right), \quad x, y \in I, x \neq y,
$$

we obtain

$$
g^{\prime}\left(\left(f^{\prime}\right)^{-1}\left(\frac{f(x)-f(y)}{x-y}\right)\right)=\frac{g(x)-g(y)}{x-y}, \quad x, y \in I, x \neq y,
$$

whence, setting

$$
\phi:=g^{\prime} \circ\left(f^{\prime}\right)^{-1}
$$

we get the equality

$$
\phi\left(\frac{f(x)-f(y)}{x-y}\right)=\frac{g(x)-g(y)}{x-y}, \quad x, y \in I, x \neq y .
$$

By the Darboux property of derivative, $f^{\prime}$ is continuous and strictly monotonic (cf. [7], Remark 2.1), so $f$ is either strictly convex or strictly concave in $I$. In view of Lemma 3.1 there exist $a, b \in \mathbb{R}$ such that

$$
\phi(u)=a u+b, \quad u \in I_{f},
$$

whence, by (3.1),

$$
a \frac{f(x)-f(y)}{x-y}+b=\frac{g(x)-g(y)}{x-y}, \quad x, y \in I, x \neq y,
$$

which implies that

$$
g(x)-a f(x)-b x=g(y)-a f(y)-b y, \quad x, y \in I .
$$

Consequently, there is $c \in \mathbb{R}$ such that

$$
g(x)=a f(x)+b x+c, \quad x \in I,
$$

which completes the proof of (i). If $g^{\prime}$ is one-to-one then, clearly, in this formula the number $a$ cannot be zero. 
Remark 3.3 Assuming the integral definition of the Lagrange mean, Berrone \& Moro proved [[1], Corollary 7] that if $f^{\prime}$ and $g^{\prime}$ be continuous and strictly monotonic functions defined on an interval $I$, then their corresponding Lagrange means coincide if, and only if, there are two real constants $\gamma_{1}, \gamma_{2}$, with $\gamma_{1} \neq 0$ such that

$$
g^{\prime}=\gamma_{1} f^{\prime}+\gamma_{2} \text { on } I .
$$

In fact, part (ii) of Theorem 3.2 is equivalent to the result of Berrone \& Moro.

\section{The homogeneity of $L^{[f]}$ implies the high regularity of $f$}

In this section we prove the following

Theorem 4.1 Let $I \subset(0, \infty)$ be an open interval. Suppose that $f: I \rightarrow \mathbb{R}$ is differentiable and $f^{\prime}$ is one-to-one. If the Lagrange mean $L^{[f]}$ is conditionally positively homogeneous, that is, if

$$
L^{[f]}(t x, t y)=t L^{[f]}(x, y)
$$

for all $x, y \in I$ and $t>0$ such that $t x, t y \in I$, then $f$ is of the class $C^{\infty}$ in $I$.

Proof Assume that $L^{[f]}$ is conditionally positively homogeneous. Then, by the definition of the Lagrange mean,

$$
\left(f^{\prime}\right)^{-1}\left(\frac{f(t x)-f(t y)}{t x-t y}\right)=t\left(f^{\prime}\right)^{-1}\left(\frac{f(x)-f(y)}{x-y}\right)
$$

for all $x, y \in I, x \neq y$, and $t>0$ such that $t x, t y \in I$. Take arbitrary $u \in I$. According to Remark 2.3 there exist $x, y \in I, x \neq y$, such that $L^{[f]}(x, y)=u$. From (3.1) we get

$$
f^{\prime}(u t)=\frac{f(t x)-f(t y)}{t x-t y} .
$$

Since the right-hand side is a differentiable function of $t$ in a neighborhood of 1 , the function $f^{\prime}$ is differentiable in a neighborhood at the point $u$. Since $u \in I$ is arbitrary, the function $f$ is twice differentiable in $I$. Now an easy inductive argument shows that $f$ is of the class $C^{\infty}$ in $I$.

\section{Equality of means as an approach to determine the homogeneous Lagrange means}

We apply Theorem 3.2 on the equality of two Lagrange means to prove the following

Theorem 5.1 Let $I \subset(0, \infty)$ be an open interval. Suppose that $f: I \rightarrow \mathbb{R}$ is differentiable and $f^{\prime}$ is one-to-one. Then the following conditions are equivalent:

(i) the Lagrange mean $L^{[f]}$ is conditionally positively homogeneous, that is,

$$
L^{[f]}(t x, t y)=t L^{[f]}(x, y)
$$

for all $x, y \in I$ and $t>0$ such that $t x, t y \in I$;

(ii) there are $p, a, b, c \in \mathbb{R}, p \neq 1, a \neq 0$, such that

$$
f(x)=\left\{\begin{array}{lll}
a x^{p}+b x+c, & x \in I & \text { if } 0 \neq p \neq 1 \\
a \ln x+b x+c, & x \in I & \text { if } p=0 \\
a x \ln x+b x+c & x \in I & \text { if } p=1
\end{array}\right.
$$


(iii) there is $p \in \mathbb{R}$ such that $L^{[f]}=\left.\mathcal{L}^{[p]}\right|_{I^{2}}$ where $\mathcal{L}^{[p]}:(0, \infty)^{2} \rightarrow(0, \infty)$ is defined by

$$
\mathcal{L}^{[p]}(x, y):=\left\{\begin{array}{ll}
\left(\frac{x^{p}-y^{p}}{p(x-y)}\right)^{1 /(p-1)} & \text { if } p \neq 0, p \neq 1 \\
\frac{x-y}{\ln x-\ln y} & \text { if } p=0 \\
e^{-1}\left(\frac{x^{x}}{y^{y}}\right)^{1 /(x-y)} & \text { if } p=1
\end{array} \quad \text { for } x \neq y ; \quad \mathcal{L}^{[p]}(x, x)=x\right.
$$

Proof To show the implication $(i) \Rightarrow$ (ii) assume $(i)$. By Theorem 4.1 the function $f$ is twice differentiable in $I$. Since $f^{\prime}$ is one-to-one, the set $\left\{x \in I: f^{\prime \prime}(x)=0\right\}$ is nowhere dense in $(0, \infty)$. Let $J \subset I$ be a maximal (in the sense of inclusion) open interval such that $f^{\prime \prime}(x) \neq 0$ for all $x \in J$. Since the interval $J$ is open, for arbitrarily fixed $x, y \in J$, $x \neq y$, there is $\delta>0$ such that for all $t \in(1-\delta, 1+\delta)$ we have $t x, t y \in J$, whence $L^{[f]}(t x, t y) \in J$ and, consequently,

$$
f^{\prime \prime}\left(L^{[f]}(t x, t y)\right) \neq 0, \quad t \in(1-\delta, 1+\delta) .
$$

Differentiating both sides of (5.1) with respect to $t \in(1-\delta, 1+\delta)$, we get

$$
\frac{\left[x f^{\prime}(t x)-y f^{\prime}(t y)\right](t x-t y)-[f(t x)-f(t y)](x-y)}{f^{\prime \prime}\left(\left(f^{\prime}\right)^{-1}\left(\frac{f(t x)-f(t y)}{t(x-y)}\right)\right)(t x-t y)^{2}}=\left(f^{\prime}\right)^{-1}\left(\frac{f(x)-f(y)}{x-y}\right),
$$

that is

$$
\frac{\left[x f^{\prime}(t x)-y f^{\prime}(t y)\right](t x-t y)-[f(t x)-f(t y)](x-y)}{f^{\prime \prime}\left(L^{[f]}(t x, t y)\right)(t x-t y)^{2}}=L^{[f]}(t x, t y),
$$

whence, setting $t=1$, after obvious simplification, we obtain

$$
\frac{\left[x f^{\prime}(x)-f(x)\right]-\left[y f^{\prime}(y)-f(y)\right]}{x-y}=L^{[f]}(x, y) f^{\prime \prime}\left(L^{[f]}(x, y)\right), \quad x, y \in J, x \neq y .
$$

Since the function $g: J \rightarrow \mathbb{R}$ defined by

$$
g(x):=x f^{\prime}(x)-f(x)
$$

is differentiable and

$$
g^{\prime}(x)=x f^{\prime \prime}(x), \quad x \in J,
$$

we can write this equality in the form

$$
\frac{g(x)-g(y)}{x-y}=g^{\prime}\left(L^{[f]}(x, y)\right), \quad x, y \in J, x \neq y,
$$

which implies that $L^{[g]}=L^{[f]}$ in $J^{2}$. By Theorem 3.2(i) there are $\alpha, \beta, \gamma \in \mathbb{R}$, such that

$$
g(x)=\alpha f(x)+\beta x+\gamma, \quad x \in J .
$$

In view of (5.3) we hence get

$$
x f^{\prime}(x)-f(x)=\alpha f(x)+\beta x+\gamma, \quad x \in J,
$$

whence, setting

$$
p:=\alpha+1
$$


we obtain

$$
x f^{\prime}(x)=p f(x)+\beta x+\gamma, \quad x \in J .
$$

Solving this linear differential equation, in the case when $0 \neq p \neq 1$, we get

$$
f(x)=a x^{p}+b x+c, \quad x \in J,
$$

in the case when $p=0$, we get

$$
f(x)=a \ln x+b x+c, \quad x \in J,
$$

and in the case when $p=1$, we get

$$
f(x)=a x \ln x+b x+c, \quad x \in J,
$$

for some $a, b, c \in \mathbb{R}$. Since, by the assumption, $f^{\prime}$ is one-to-one, we conclude that the number $a$ cannot be zero. Note also that in the first case

$$
f^{\prime \prime}(x)=p(p-1) a x^{p-2}, \quad x \in J,
$$

in the second case when $p=0$,

$$
f^{\prime \prime}(x)=-\frac{a}{x^{2}}, \quad x \in J,
$$

and, in the case $p=1$,

$$
f^{\prime \prime}(x)=\frac{a}{x}, \quad x \in J .
$$

Since $J$ is a maximal subinterval of $I$ such that $f^{\prime \prime}(x) \neq 0$ for all $x \in J$, it follows that $J=I$ for all $p$. This proves that $(i i)$ holds true.

Since the remaining implications $(i i) \Rightarrow($ iii $)$ and $(i i i) \Rightarrow(i)$ are easy to verify, the proof is complete.

Remark 5.2 Clearly, for any $p \in \mathbb{R}$, the mean $\mathcal{L}^{[p]}$ is posiyively homogeneous, that is

$$
\mathcal{L}^{[p]}(t x, t y)=t \mathcal{L}^{[p]}(x, y), \quad t, x, y>0 .
$$

The mean $\mathcal{L}^{[0]}$ is called the logarithmic mean, the mean $\mathcal{L}^{[1]}$, defined as the pointwise limit, by the formula

$$
\mathcal{L}^{[1]}(x, y):=\lim _{p \rightarrow 1} \mathcal{L}^{[p]}(x, y), \quad x, y>0,
$$

is called the identric mean, and $\left\{\mathcal{L}^{[p]}: p \in \mathbb{R}\right\}$ is sometime called the family of generalized logarithmic means. It is well-know (and obvious) that, for arbitrary $(x, y) \in(0, \infty)^{2}$, the function

$$
\mathbb{R} \ni p \rightarrow \mathcal{L}^{[p]}(x, y)
$$

is continuous.

\section{Remarks on the relevant question for Cauchy means}

Let $I \subset \mathbb{R}$ be an interval. Assume that $f, g: I \rightarrow \mathbb{R}$ are differentiable and $g^{\prime}(x) \neq 0$ for all $x \in I$. By the Cauchy mean-value theorem, there exists a Cauchy mean $C^{[f, g]}: I^{2} \rightarrow I$ such that, for all $x, y \in I, x \neq y$,

$$
\frac{f(x)-f(y)}{g(x)-g(y)}=\frac{f^{\prime}}{g^{\prime}}\left(C^{[f, g]}(x, y)\right) .
$$


(The functions $f$ and $g$ are called the generators of $C^{[f, g]}$.) If moreover $\frac{f^{\prime}}{g^{\prime}}$ is one-to-one, then

$$
C^{[f, g]}(x, y)=\left\{\begin{array}{ll}
\left(\frac{f^{\prime}}{g^{\prime}}\right)^{-1}\left(\frac{f(x)-f(y)}{g(x)-g(y)}\right) & \text { for } x \neq y \\
x & \text { for } x=y
\end{array},\right.
$$

so $C^{[f, g]}$ is uniquely determined. Note here that

$$
\frac{f^{\prime}}{g^{\prime}} \circ g^{-1}=\left(f \circ g^{-1}\right)^{\prime} \quad \text { or, equivalently, } \quad \frac{f^{\prime}}{g^{\prime}}=\left(f \circ g^{-1}\right) \circ g .
$$

In [7], Proposition 1, it was observed that

$$
C^{[f, g]}(x, y)=g^{-1}\left(L^{\left[f \circ g^{-1}\right]}(g(x), g(y))\right), \quad x \cdot y \in I,
$$

that is the Cauchy mean $C^{[f, g]}$ is $g$-conjugate to the Lagrange mean $L^{\left[f \circ g^{-1}\right]}$ of the generator $f \circ g^{-1}$. Hence we have

$$
L^{\left[f \circ g^{-1}\right]}(u, v)=g\left(C^{[f, g]}\left(g^{-1}(u), g^{-1}(v)\right)\right), \quad u, v \in g(I) .
$$

Now applying Theorem 5.1 with $f$ replaced by $f \circ g^{-1}$ we obtain the following

Corollary 6.1 Let $I \subset(0, \infty)$ be an open interval. Suppose that $f, g: I \rightarrow \mathbb{R}$ are differentiable, $g^{\prime}(x) \neq 0$ for all $x \in I$, and $\left(\frac{f^{\prime}}{g^{\prime}}\right)^{-1}$ is one-to-one. Then the following conditions are equivalent:

(i) the Lagrange mean $L^{\left[f \circ g^{-1}\right]}$ is conditionally positively homogeneous, that is,

$$
L^{\left[f \circ g^{-1}\right]}(t u, t v)=t L^{\left[f \circ g^{-1}\right]}(u, v)
$$

for all $u, v \in I$ and $t>0$ such that $t u, t v \in g(I)$;

(ii) there are $p, a, b, c \in \mathbb{R}, p \neq 1, a \neq 0$, such that

$$
f(x)=\left\{\begin{array}{lll}
a[g(x)]^{p}+b g(x)+c, & x \in I & \text { if } p \neq 0 \\
a \ln g(x)+b(x)+c, & x \in I & \text { if } p=0
\end{array}\right.
$$

(iii) there is $p \in \mathbb{R}, p \neq 1$, such that

$$
C^{[f, g]}(x, y)=g^{-1}\left(\left.\mathcal{L}^{[p]}\right|_{g(I)^{2}}(g(x), g(y))\right), \quad x, y \in I,
$$

where $\mathcal{L}^{[p]}:(0, \infty)^{2} \rightarrow(0, \infty)$, for every $p \in \mathbb{R}$, is defined by (5.2).

In this context it is natural to ask if we can determine all conditionally positively homogeneous Cauchy means by a similar method as that in the case of Lagrange means. It turns out that, in general not. First of all let us note that, under the assumptions of the definition, the condition

$$
C^{[f, g]}(t x, t y)=t C^{[f, g]}(x, y)
$$

for all $x, y \in I$ and $t>0$ such that $t x, t y \in I$, does not imply that both $f$ and $g$ must be of the higher regularity. It is not difficult to see that this condition and the assumption that one of the functions of $f$ or $g$ is twice differentiable imply that the remaining one is also twice differentiable.

Assuming that one of these functions is twice differentiable and differentiating both sides of (6.1) with respect to $t$ and then setting $t=1$ leads to the following interesting equality 


$$
\frac{C^{[f, g]}(x, y) \cdot\left(\frac{f^{\prime}}{g^{\prime}}\right)^{\prime}\left(C^{[f, g]}(x, y)\right)}{\frac{f^{\prime}}{g^{\prime}}\left(C^{[f, g]}(x, y)\right)}=\frac{x f^{\prime}(x)-y f^{\prime}(y)}{f(x)-f(y)}-\frac{x g^{\prime}(x)-y g^{\prime}(y)}{g(x)-g(y)}
$$

for all $x, y \in I, x \neq y$. Hence, setting

$$
\varphi_{h}(u):=\frac{u h^{\prime}(u)}{h(u)},
$$

for a differentiable function $h$ such that $h^{\prime}(u) \neq 0$ for all $u \in I$, and applying the Cauchy mean-value theorem for each of two difference quotient on the right side, we obtain

$$
\varphi_{\frac{f^{\prime}}{g^{\prime}}}\left(C^{[f, g]}(x, y)\right)=\varphi_{f}\left(C^{[f, \text { id } \cdot f]}(x, y)\right)-\varphi_{g}\left(C^{[g, \text { id } \cdot g]}(x, y)\right), \quad x, y \in I .
$$

Thus we have shown the following

Corollary 6.2 Let $I \subset(0, \infty)$ be an open interval. Suppose that $f, g: I \rightarrow \mathbb{R}$ are differentiable, $g^{\prime}(x) \neq 0$ for all $x \in I$, and $\left(\frac{f^{\prime}}{g^{\prime}}\right)^{-1}$ is one-to-one. If the Cauchy mean $C^{[f, g]}$ is conditionally positively homogeneous, then (6.2) holds true.

Open Access This article is distributed under the terms of the Creative Commons Attribution License which permits any use, distribution, and reproduction in any medium, provided the original author(s) and the source are credited.

\section{References}

1. L.R. Berrone, J. Moro, Lagrangian means. Aequationes Math. 55(5), 217-226 (1998)

2. P.S. Bullen, D.S. Mitrinović, P.M. Vasić, Means and their inequalities, mathematics and its applications (D. Reidel Publishing Company, Dodrecht-Boston-Lancaster-Tokyo, 1988)

3. P.S. Bullen, Handbook of means and their inequalities, mathematics and its applications, vol. 560 (Kluwer Academic Publishers, Dordrecht/ Boston/ London, 2003)

4. D. Głazowska, An invariance of the geometric meanwith respect to Lagrangian Conditionally homogeneous mean-type mappings. Demonstr. Math. 20(2), 289-302 (2007)

5. B. Jessen, Bemœarkninger om konvekse Funktioner og Uligheder imellen Midelvœdier I, II, Mat. Tidsskr., B (1931b), 17-28, 84-95

6. J. Matkowski, Mean value property and associated functional equation. Aequationes Math. 58, 46-59 (1999)

7. J. Matkowski, On weighted extensions of Cauchy’s means. J. Math. Anal. Appl. 319, 215-227 (2006) 